86 CARDIAC AUTONOMIC FUNCTION INFLUENCES IN ATRIAL FIBRILLATION AND HYPERTENSION

${ }^{1}$ Ahsan Khan, ${ }^{2}$ Rehan Junejo, ${ }^{3}$ James Fisher, ${ }^{4}$ Neil Thomas, ${ }^{5} \mathrm{G}$. Lip. ${ }^{1}$ University of Birmingham, Birmingham, UK; ${ }^{2}$ Manchester Metropolitan University; ${ }^{3}$ University of Auckland; ${ }^{4}$ Institute of Applied Health Research, University of Birmingham; ${ }^{5}$ Liverpool Centre for Cardiovascular Science, University of Liverpool and Liverpool Heart \& Chest

\subsection{6/heartjnl-2021-BCS.86}

Introduction Atrial fibrillation (AF) is widely recognised as a significant cardiovascular condition associated with poor outcomes. There is increasing evidence that abnormalities of the cardiac autonomic nervous system (ANS) are involved in the pathogenesis of AF. AF and hypertension commonly co-exist and are independently associated with impaired autonomic function determined using heart rate variability (HRV). We decided to investigate whether HRV is more abnormal in patients with AF and hypertension when compared to hypertension alone.

Methods In a cross-sectional comparison, we studied two patient groups: AF and hypertension $(\mathrm{n}=61)$ and hypertension control group $(\mathrm{n}=33)$. Time-domain, frequency-domain and non-linear measures of HRV were determined using eMotion Faros ECG sensor. Participant's breathing was controlled with a metronome. Data was analysed using SPSS software.

Results Participants were matched for age, sex and body mass index (BMI). Time-domain and non-linear indices of HRV were higher in AF (and hypertension) group compared to hypertensive controls $(p \leq 0.01)$ (table 1$)$. AF $(p=0.003)$, ejection fraction $(p=0.04)$ and heart rate $(p=0.04)$ were independently associated with changes seen on HRV following adjustment for multiple variables.

\begin{tabular}{|c|c|c|c|}
\hline & $\begin{array}{l}\text { AF }+ \text { hypertension group } \\
(\mathrm{n}=40)\end{array}$ & $\begin{array}{l}\text { Hypertension control } \\
\text { group } \\
(n=20)\end{array}$ & $\mathrm{p}$ \\
\hline $\begin{array}{l}\text { Clinical } \\
\text { Demographics }\end{array}$ & Mean \pm SD / Median [IQR] & Mean \pm SD / Median [IQR] & \\
\hline Age, years & $66 \pm 7$ & $65 \pm 7$ & 0.71 \\
\hline \multicolumn{4}{|l|}{ Sex } \\
\hline Male & 29 & 15 & 0.84 \\
\hline Female & 11 & 5 & \\
\hline BMI $\left(\mathrm{kg} / \mathrm{m}^{2}\right)$ & $32.9 \pm 5.2$ & $32.1 \pm 4.2$ & 0.58 \\
\hline $\begin{array}{l}\text { HRV } \\
\text { Measurements }\end{array}$ & $\begin{array}{l}\text { Mean }[95 \% \mathrm{Cl}] / \text { Median } \\
{[95 \% \mathrm{Cl}]}\end{array}$ & $\begin{array}{l}\text { Mean }[95 \% \mathrm{Cl}] / \text { Median } \\
{[95 \% \mathrm{Cl}]}\end{array}$ & $\mathrm{p}$ \\
\hline Heart rate & $66[61-72]^{\mathrm{a}}$ & $60[56-66]^{a}$ & 0.12 \\
\hline SDNN (ms) & $62[48-80]^{a}$ & $33[27-41]^{a}$ & $<0.001$ \\
\hline rMSSD (ms) & $97[35-105]^{b}$ & $26[20-37]^{b}$ & 0.002 \\
\hline pNN50 (\%) & $58[25-64]^{b}$ & $5[1-17]^{b}$ & $<0.001$ \\
\hline LF normalised (\%) & $42[33-52]^{\mathrm{a}^{*}}$ & $48[38-58]^{\mathrm{a}}$ & 0.39 \\
\hline HF normalised (\%) & $58[48-67]^{\mathrm{a}^{*}}$ & $52[42-62]^{a}$ & 0.39 \\
\hline LF-i/HF-i & $0.7[0.5-1.1]^{\mathrm{a}^{\star}}$ & $0.8[0.6-1.2]^{\mathrm{a}}$ & 0.61 \\
\hline SD1 (ms) & $65[38-74]^{b}$ & $18[14-26]^{b}$ & $<0.001$ \\
\hline SD2 (ms) & $90[72-108]^{\mathrm{a}}$ & $47[36-58]^{\mathrm{a}}$ & $<0.001$ \\
\hline SD1/SD2 & $0.7[0.6-0.7]^{a}$ & $0.5[0.4-0.5]^{\mathrm{a}}$ & 0.002 \\
\hline
\end{tabular}

Normally distributed data are expressed as mean \pm standard deviation for descriptive data and mean $[95 \%$ confidence interval $(\mathrm{CI})]$ otherwise. Identified bysuperscript a. Non-normally distributed data are displayed as median withinterquartile ranges for descriptive data and median $[95 \% \mathrm{Cl}]$ otherwise.Identified by superscript b. Normality test was performed using Shapiro-Wilktest. Statistical differences were tested usingindependent t-test (for parametric data) or Mann-Whitney $U$ test (fornon-parametric data). Significance $p \geq 0.05$.
Conclusions First study investigating autonomic function in patients with permanent $\mathrm{AF}$ and hypertension. AF, independent of hypertension, is characterised with marked HRV and is possibly related to vagal tone.

Conflict of Interest None

\section{ADAPTING ATRIAL FIBRILLATION ABLATION TO COVID TIMES: THE FEASIBILITY OF VERY HIGH POWER SHORT DURATION ABLATION UNDER MILD CONSCIOUS SEDATION}

${ }^{1}$ Gavin Chu, ${ }^{2}$ Bharat Sidhu, ${ }^{2}$ Akash Mavilakandy, ${ }^{1}$ Vishal Luther, ${ }^{1}$ Richard Snowdon, ${ }^{2}$ Andre Ng, 'DHIRAJ GUPTA. 'Liverpool Heart and Chest Hospital NHS Foundation Trust, Liverpool, UK; ${ }^{2}$ University of Leicester

\subsection{6/heartjnl-2021-BCS.87}

Introduction Radiofrequency (RF) ablation for atrial fibrillation (AF) has traditionally been performed under general anaesthesia (GA) to improve procedure tolerance and efficacy, but this has been compromised during the COVID-19 pandemic due to a reduction in GA availability. A very high-power short duration (vHPSD) energy delivery protocol may reduce RF delivery times and hence overall procedure duration, potentially obviating the need for GA when using such an approach. However, the use of vHPSD under conscious sedation has not previously been reported.

We sought to evaluate first-time $\mathrm{AF}$ ablation using a vHPSD approach during the COVID-19 pandemic by comparing the procedural metrics and same day discharge (SDD) rates of vHPSD against cryoballoon ablation.

Methods Procedural data was collected from consecutive patients undergoing first-time $\mathrm{AF}$ ablation at two UK centres from September 2020 to February 2021 using either the QDot Micro catheter (Biosense Webster) or the Arctic Front Advance Pro cryoballoon (Medtronic). In the QDot group, vHPSD ablation (90W, 4 second lesions) was mandated for pulmonary vein isolation (PVI), while Ablation-Index guided 50W ablation was allowed for additional lesions. Procedures were performed under mild conscious sedation with opiates and benzodiazepines, with a default strategy of SDD in the absence of clinical concerns or adverse events.

Results 78 patients were evaluated, with 39 patients undergoing vHPSD and 39 receiving cryoablation. The procedural metrics of both groups are shown in the table 1. 34 out of $39(87 \%)$ vHPSD procedures were under conscious sedation, and the 5 GA cases were all from the initial 2 months of experience with the Qdot catheter. The duration of RF energy delivery to achieve PVI using vHPSD was significantly shorter than the equivalent duration of cryothermy. Overall fluoroscopy times were shorter using vHPSD, while procedure duration was longer. There was failure to achieve isolation of all pulmonary veins in $3(7.7 \%)$ cryoablation patients versus none when using vHPSD ablation. In the vHPSD group. 3 patients received adjunctive ablation beyond PVI: 1 had roof and floor lines; 1 cavotricuspid isthmus line, and 1 received a mitral isthmus line. No adjunctive ablation was performed in the cryoablation group. SDD rates were similar in in both groups.

Conclusion A vHPSD approach can be used with conscious sedation to achieve same-day discharge rates for AF ablation that are comparable to cryoablation. There are advantages in fluoroscopy time and the required duration of ablation delivery, as well as the versatility to handle variations in 